\title{
Phenotyping for Waterlogging Tolerance as a Proxy for Phytophthora medicaginis Resistance in Chickpea
}

\author{
Nicole Dron, ${ }^{1,+}$ Tim Sutton, ${ }^{2,3}$ Steve Harden, ${ }^{1}$ Steven Simpfendorfer, ${ }^{1}$ and Kristy Hobson ${ }^{1}$ \\ ${ }^{1}$ NSW Department of Primary Industries, 4 Marsden Park Road, Tamworth, NSW 2340, Australia \\ ${ }^{2}$ South Australian Research and Development Institute, GPO Box 397, Adelaide, SA 5001, Australia \\ ${ }^{3}$ School of Agriculture, Food and Wine, University of Adelaide, PMB 1, Glen Osmond, SA 5064, Australia
}

Accepted for publication 30 May 2021.

\begin{abstract}
Phytophthora root rot (PRR) caused by the soilborne oomycete Phytophthora medicaginis is a significant constraint to chickpea (Cicer arietinum) production across the northern grains region of Australia. In flooded soil, which is conducive to PRR disease development, up to $70 \%$ yield loss can occur in the most resistant Australian cultivars. Incorporating waterlogging tolerance in soybean (Glycine max) has been shown to improve quantitative resistance to Phytophthora sojae. Root growth of three chickpea genotypes was assessed at the seedling stage under waterlogging, PRR, and the combination of these abiotic and biotic constraints. Levels of waterlogging tolerance in chickpea are inherently low, yet selected genotypes displayed variability in root traits linked to improved waterlogging tolerance. The PRR moderately susceptible chickpea cultivar Yorker and PRR very susceptible Rupali demonstrated an eightfold increase in early adventitious root growth from the epicotyl region under waterlogging stress, compared with the PRR resistant
\end{abstract}

Abstrac

\section{Background}

Chickpea (Cicer arietinum) is an important global source of plant protein within human diets and a valuable rotation crop in broadacre systems. Countries including India, Pakistan, Turkey, Iran, Myanmar, Iraq, Ethiopia, Mexico, Spain, Canada, and Australia are major producers of chickpea (Boye et al. 2010; Knights et al. 2007). Chickpea was first introduced into Australian cropping systems in the early 1980s; production peaked in 2017 with 1.1 million hectares sown in response to rising export prices. Over $70 \%$ of the area sown to chickpea is located in the northern grains region (northern New South Wales and southern Queensland) (ABARES 2018). Phytophthora root rot (PRR) caused by the oomycete Phytophthora medicaginis (E. M. Hansen and D. P. Maxwell) is prevalent across this northern grains region, with yield losses estimated at AUD 8.2 million annually (Murray and Brennan 2012). Various Phytophthora spp. have been associated with PRR of

†Corresponding author: N. Dron; nicole.dron@dpi.nsw.gov.au

Funding: This research and Ph.D. candidate Nicole Dron were supported by the Grains Research and Development Corporation and New South Wales Department of Primary Industries through the project BLG302. The student's study was supported through the University of Adelaide.

The author(s) declare no conflict of interest.

(c) 2021 The American Phytopathological Society interspecific backcross genotype 04067-81-2-1-1 (C. echinospermum $\times$ C. arietinum*2). Selection for primary root depth, which was significantly greater in 04067-81-2-1-1 under waterlogging, appears to improve PRR resistance compared with root replacement traits. Soilborne Phytophthora spp. are reportedly attracted to branch sites and leached exudates. We propose that compromised root barriers at emergence sites of adventitious roots under waterlogging conditions hasten hyphal entry, potentially increasing susceptibility to PRR. Hence, screening for root depth and absence of adventitious root development under waterlogged conditions may offer a novel proxy phenotyping method for PRR resistance traits at early stages of chickpea breeding.

Keywords: chickpea, rapid phenotyping, resistance breeding, Phytophthora medicaginis, waterlogging tolerance, root morphology, adventitious roots chickpea crops across parts of Argentina, India, Pakistan, and Spain, but they are of considerably lower economic impact compared with in Australia (Nene et al. 1996).

Flooding has been identified as an important precondition to severe PRR development (Kuan and Erwin 1980; Schwinghamer et al. 2011). Extreme weather events, including flooding, are predicted to increase globally as a result of climate change, so understanding the links between PRR and waterlogging is becoming increasingly relevant (Arnell 2004; Hirabayashi et al. 2013). Under dryland conditions in the northern region of Australia, yield losses of 30 to $50 \%$ are typical for the moderately susceptible PRR chickpea cultivar Yorker, yet under extended periods of waterlogging up to $70 \%$ yield loss from PRR has been recorded in this cultivar (Bithell et al. 2021). There are two established concepts that explain the increase in PRR pressure under waterlogged conditions: an increase in Phytophthora inoculum (Erwin et al. 1983; Pfender et al. 1977) and a breakdown of plant resistance due to physiological damage, restricted metabolic function, and reduced capacity to overcome stress (Jackson and Colmer 2005; Stolzy and Sojka 1984). In soybean (Glycine max), four chromosomal regions associated with both waterlogging tolerance and $P$. sojae resistance additively reduced yield losses from PRR (Cornelious et al. 2005; Nguyen et al. 2012). Therefore, it is likely that incorporating waterlogging tolerance into chickpea could improve the durability of resistance to $P$. medicaginis under high soil moisture conditions favorable to the development of PRR. 
Disease pressure induced by Phytophthora spp. is directly associated with their polycyclic lifecycle, which is driven by environmental conditions. In drier environments, the homothallic $P$. medicaginis oospores undergo direct germination resulting in localized root infections, causing low inoculum pressure. In environments with greater moisture, oospores germinate rapidly, favoring the production of sporangia, which release large numbers of motile zoospores, initiating multiple root infections and resulting in high inoculum pressure (Erwin et al. 1983; Pfender et al. 1977; Van West et al. 2003). In the field, symptoms of PRR include a reduction or cessation of growth rate, leaf chlorosis, desiccation of foliage, premature senescence, wilting, decay of the lateral roots, reddish-brown stem canker, and yield loss. Symptoms of waterlogging are similar to PRR, with the exclusion of characteristic stem canker and root lesions (Chen et al. 2011; Erwin et al. 1983).

Phytophthora inoculum in soil has been reported to survive up to 5 years after a host crop, so rotation with alternative nonhost crops is largely impractical and ineffective (Erwin et al. 1983). Application of fungicides, such as short-acting metalaxyl, has been shown to reduce PRR, but they are uneconomical at the broadacre scale. Hence, selection of improved levels of PRR resistance remains a high priority for chickpea breeding in Australia. Sources of PRR resistance within the cultivated chickpea species $C$. arietinum are scarce. Moderate levels of resistance have been incorporated from a landrace (ICC11870) into the cultivars Yorker, PBA HatTrick, and PBA Seamer (Brinsmead et al. 1985). Wild Cicer accessions (C. echinospermum P.H. Davis and C. reticulatum Ladizinsky) offer high levels of resistance but present major challenges for breeding because of the prostrate growth habit affecting harvestability, low yields, and poor seed quality (Knights et al. 2008). Linkage drag of agronomically undesirable characteristics is therefore a major challenge when introgressing PRR resistance loci from these wild Cicer sources. High levels of waterlogging tolerance in chickpea have not yet been found (Chauhan 1987; Cowie et al. 1995; Palta et al. 2010). However, waterlogging tolerance may have been indirectly selected for in the Australian breeding strategy through efforts to increase PRR resistance.

PRR resistance in chickpea is predominantly identified through field screening and selection, where the process is highly dependent on achieving the required soil moisture for plant and disease establishment. More so, field heterogeneity generates spatial variability in disease pressure, making it difficult to uncouple genotypic and environmental effects. In order to overcome this problem, breeding programs have successfully utilized controlled-environment phenotyping systems in parallel to field screening. Greenhouse soil-based and hydroponic methods have been developed in which seedlings are inoculated using a $P$. medicaginis mycelial-oospore and zoospore inoculum (Amalraj et al. 2019b; Knights et al. 2008). Both field and controlled environments were able to identify the best sources of PRR resistance, although in some cases genotypes with moderate resistance shuffled in their resistance rankings (Amalraj et al. 2019a; Knights et al. 2008). Alternatively, phenotyping for PRR resistance in a wet environment (e.g., hydroponics or under flooding in the field) and focusing on specific root traits closely associated with waterlogging tolerance may identify additional genetic regions associated with higher levels of genetic resistance to PRR. Furthermore, less destructive and more repeatable waterlogging phenotyping methods could be used as a proxy for PRR resistance selection within early generation material, significantly increasing genetic gain in breeding programs.
The objective of this study was to determine if root traits related to waterlogging tolerance could be identified in chickpea using a rapid waterlogging phenotyping method and investigate whether these traits are associated with improved resistance to PRR. We describe a novel rapid waterlogging phenotyping method and measurable root traits of chickpea that could be used to select for improved resistance to PRR.

\section{Materials and Methods}

Plant and oomycete material. Three chickpea lines that differ in PRR resistance were used to develop the controlledenvironment phenotyping protocols (Amalraj et al. 2019a). The PRR-resistant wild $C$. echinospermum backcross (BC) genotype 04067-81-2-1-1 (pedigree: Howzat/ILWC 245//99039-1013), the moderately susceptible $C$. arietinum Yorker (pedigree: 850728H/946-31), and the very susceptible Rupali (pedigree: FLIP8415C/ICCV88516//Amethyst).

The $P$. medicaginis isolate TR4046 used in this study was recovered from a PRR-infected chickpea plant near Moree, New South Wales, in 2005. The isolate was cultured on V8 agar with $2.5 \%$ $\mathrm{CaCO}_{3}$ for 4 weeks at $25^{\circ} \mathrm{C}$ in darkness until mycelium had spread across the plate and oospores within the agar had matured. Mycelial mats and agar were flooded with reverse osmosis (RO) water and macerated for $130 \mathrm{~s}$ with a stick blender. Oospores in the resulting liquid mycelial-oospore suspension were counted using a hemocytometer.

Experimental design and conditions. This study comprised two experiments (experiment 1 and experiment 2) with both conducted in a glasshouse located at the Tamworth Agricultural Institute in Tamworth, New South Wales. Diurnal temperatures were set to $25^{\circ} \mathrm{C} / 18^{\circ} \mathrm{C}$; a higher temperature range was selected to accelerate waterlogging damage to the seedlings (Cowie et al. 1995). Experiment 1 was conducted during summer and experiment 2 in the winter months of 2018. A split-plot design was used with waterlogging $\times P$. medicaginis inoculation at the main plot treatment level and chickpea lines the subplot treatment level. Main treatments included a control, P. medicaginis-inoculated (PRR), waterlogging (WL), and the combination of both waterlogging and $P$. medicaginis inoculation (PRR:WL). There were six replicates of each main treatment combination. Sixteen seedling tubes were held in place in a plastic container $(22 \times 22 \times 12 \mathrm{~cm})$ with five drainage holes; this container was inserted into a second fully sealed plastic container of the same size to hold water within waterlogging treatments (Fig. 1).

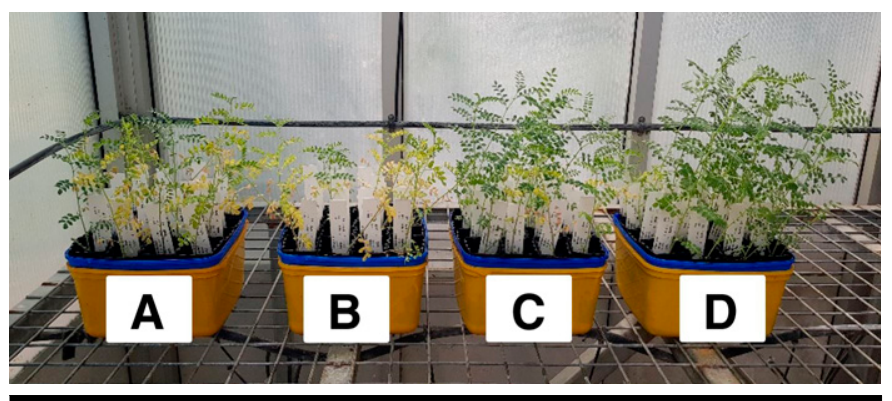

\section{FIGURE 1}

Growth of chickpea plants at 12 days following four treatments: $\mathbf{A}$, inoculated with Phytophthora medicaginis only; B, inoculated with $P$. medicaginis and subjected to waterlogging; $\mathbf{C}$, subjected to waterlogging only; and $\mathbf{D}$, control (no inoculation and no waterlogging). 
In experiment 1 , five or six tubes of the three chickpea genotypes (04067-81-2-1-1, Yorker, and Rupali) were randomized and held in each treatment container. Experiment 2 had a similar design, except 13 advanced chickpea breeding genotypes were evaluated alongside the three genotypes examined in experiment 1. Data from the advanced breeding genotypes were used in the analysis of experiment 2 but are not presented in this study due to being commercial in confidence.

In planta flooding and infection. Chickpea seeds were washed in $0.01 \%$ (w/v) sodium hypochlorite for $2 \mathrm{~min}$ and triple rinsed in RO water. A covered 10-liter container of 0.1-strength nutrient solution was used to germinate the seedlings on mesh. The composition of the nutrient solution in $\mathrm{RO}$ water at full strength was as follows $(\mathrm{mM}): 5.0 \mathrm{Ca}^{2+}, 5.0 \mathrm{~K}+, 0.625 \mathrm{NH}^{4+}, 0.4$ $\mathrm{Mg}^{2+}, 0.2 \mathrm{Na}^{+}, 5.4 \mathrm{SO}_{4}{ }^{2-}, 4.4 \mathrm{NO}^{3-}, 0.2 \mathrm{H}_{2} \mathrm{PO}^{4-}, 0.1 \mathrm{SiO}_{3}{ }^{2-}, 0.1$ Fe (Sequestrene), $0.05 \mathrm{Cl}^{-}, 0.025 \mathrm{BO}_{3}{ }^{3-}, 0.002 \mathrm{Mn}^{2+}, 0.002 \mathrm{Zn}^{2+}$, $0.0005 \mathrm{Cu}^{2+}, 0.0005 \mathrm{MoO}_{4}{ }^{2-}$, and $0.001 \mathrm{Ni}^{2+}$. The solution was buffered with $1.0 \mathrm{mM} 2$-( $N$-morpholino) ethane sulfonic acid (Samineni et al. 2011). After 3 days in darkness, seedlings of the same size were transplanted into free-draining potting tubes $(50 \times 50 \times 100$ $\mathrm{mm}$ ) containing $130 \mathrm{~g}$ of premoistened sieved (5-mm) potting medium (1:1:1 loam, sand, and Greenlife potting mix). Following planting, each seedling received $30 \mathrm{ml}$ of RO water every 2 to 3 days.

Treatments were applied to the seedlings at the two-leaf stage. First, $P$. medicaginis inoculum was applied by the flood application of mycelial-oospore suspension at a concentration of 30 oospores/ml in RO water. This rate was chosen because field screening showed that 1,500 oospores per plant differentiated levels of PRR resistance and was calculated based on the 2.5 liters of water applied at plant inoculation across treatments. Flooding on the surface of the potting medium remained for $48 \mathrm{~h}$. During this time all treatments, including the control, were flooded and then drained to regulate leaching of nutrients and $P$. medicaginis inoculum between waterlogged and nonwaterlogged treatments. After $1 \mathrm{~h}$ of free draining, waterlogging treatments were then refilled with RO water level with the surface of the potting medium, and flooding was maintained daily for 12 days in experiment 1 and for 15 days in experiment 2. Experimental treatments were extended in experiment 2 because the visual PRR symptoms were not evident after the 10 days, due to the cooler winter conditions and shortened day length in the glasshouse. Watering continued in nonwaterlogged treatments with $30 \mathrm{ml}$ of RO water added to the surface of the potting medium every 2 to 3 days and allowed to freely drain.

All plants were individually assessed after completion of waterlogging treatments (12 days for experiment 1 and 15 days for experiment 2). Chlorophyll fluorescence ratio (CFR) readings were taken using a handheld chlorophyll content meter (CCM300, Opti-sciences, U.S.A.). CFR is the ratio of chlorophyll fluorescence at $735 \mathrm{~nm} / 700 \mathrm{~nm}$ and is linearly proportional to chlorophyll content $\left(R^{2} \geq 0.95\right)$ (Gitelson et al. 1999). One measurement per plant was taken in the middle of the youngest fully expanded leaflet on the leaf closest to the stem at a temperature of $23 \pm$ $5^{\circ} \mathrm{C}$ for experiment 1 and $20 \pm 5^{\circ} \mathrm{C}$ for experiment 2 in the glasshouse. Plants were then removed from potting tubes and lightly washed to remove potting mix from the roots prior to measuring adventitious root counts (AR) and primary root length (PRL). AR count data were collected from the epicotyl and hypocotyl regions of chickpea seedlings. Finally, dry shoot weights (DSW) and dry root weights (DRW), with the cotyledon detached, were recorded for each seedling after drying at $60^{\circ} \mathrm{C}$ for $72 \mathrm{~h}$.
Statistical analysis. For experiment 1, a principal component analysis (PCA) and cluster analysis were performed using the partitioning around medoids (PAM) package for R (Maechler et al. 2019) to look at the association between the measured variables and grouping of the data. Most data (DRW, DSW, PRL, and CFR) were analyzed with a linear mixed model using the statistical software asreml (Butler 2020). The fixed terms in the model were the factors treatment (control, PRR, WL, and PRR:WL), genotype (three genotypes for experiment 1 and 16 genotypes for experiment 2 ), and their interaction. Random terms in the model were replicate and container within replicate. For count data (AR), a generalized linear mixed model with a "log" link was used. Least significant differences at the 5\% level and predicted means for the three lines used in both experiments were calculated using the "predictPlus" command from the R package asremlPlus (Brien 2020).

\section{Results}

The PCA (experiment 1) showed four of the phenotyping data variables (PRL, DRW, DSW, and CFR) to be positively correlated. The AR variable had low correlation with the other four. Treatments clustered in three groups, which represented the control, WL, and PRR:WL treatments (Fig. 2, left panel). The PRR treatment demonstrated greater spread across measured traits, as expected due to the underlying differences in the levels of PRR resistance between genotypes. Interestingly, the WL-only cluster separated based on AR, in which $C$. arietinum genotypes Yorker and Rupali outperformed the C. echinospermum BC 04067-81-21-1 (Fig. 2, right panel). A tight cluster was observed for the PRR:WL treatment with little genotypic effect between lines except for the PRL trait.

PRR resistance groups were reflected in DRW, DSW, PRL, and CFR in the resistant C. echinospermum BC 04067-81-2-1-1, moderately susceptible Yorker, and very susceptible Rupali in the PRR treatment when compared with the control in experiment 1 (Table 1). The PRR treatment in experiment 2 demonstrated a reduced disease severity; therefore, significant differences in DRW, DSW, PRL, and CFR across lines were not observed.

Under WL conditions, a two- to threefold reduction in DRW was observed in both experiments, across all three genotypes, even though there were significant differences in PRL and AR counts (Table 1, Fig. 3). In experiment 1 only, C. echinospermum BC 04067-81-2-1-1 maintained an average PRL of $92.0 \mathrm{~mm}$, whereas Yorker and Rupali PRL were significantly shorter at 69.0 and 62.7 $\mathrm{mm}$, respectively (Table 1). WL treatment resulted in a greater AR proliferation in Yorker and Rupali compared with 04067-81-2-1-1 in both experiments (Table 1, Fig. 3). DSW and CFR in the WL treatment did not differ significantly from the control in experiment 1 . Reductions were observed in experiment 2, perhaps due to greater duration of treatment application (Table 1).

In both experiments, the PRR:WL treatment resulted in severe root disease development and reductions in all measured traits across the three genotypes (Table 1). Plant death occurred in a small number of susceptible Rupali seedlings in both experiments. As observed in the WL treatment, 04067-81-2-1-1 (69.8 mm) maintained a greater average PRL over Yorker $(51.6 \mathrm{~mm})$ and Rupali $(53.8 \mathrm{~mm})$ in experiment 1 (Table 1). The PRR:WL treatment resulted in a significant reduction of $\mathrm{AR}$ in Yorker and Rupali lines when compared with the WL treatment, in experiment 1 (Table 1). Under the higher disease pressure in experiment 1 , the combination of PRR and WL resulted in severe rotting of AR roots. Similar trends were observed in experiment 2, in which 
Yorker and Rupali demonstrated a reduction in AR when compared with the WL treatment.

\section{Discussion}

Waterlogging had a significant effect on chickpea root morphology, which exacerbated PRR severity. Root plasticity of the three chickpea genotypes that differed in PRR resistance was examined and demonstrated significant differences in both PRL and AR branching at the seedling stage under waterlogged conditions. This suggests that chickpea PRR resistance and waterlogging tolerance could be linked, as previously reported in soybean. Further investigation is currently underway using a recombinant inbred line population.

Amalraj et al. (2019a) previously assessed levels of PRR resistance of the chickpea genotypes used in this study under field conditions. Findings showed that C. echinospermum BC 0406781-2-1-1 exhibited improved levels of PRR resistance across two varying seasons, including a high-rainfall (i.e., disease-conducive) season, whereas the moderately susceptible Yorker and highly susceptible Rupali showed a greater range in PRR plant survival (Amalraj et al. 2019a). In this study, BC 04067-81-2-1-1 demonstrated lower DRW losses than Yorker and Rupali, respectively, under PRR infection. Furthermore, BC 04067-81-2-1-1 showed improved CFR over Yorker and Rupali in both PRR and PRR:WL treatments, demonstrating that BC 04067-81-2-1-1 also had improved PRR resistance under both phenotyping conditions.

Chlorophyll content and the associated CFR are often used to phenotype stress responses including waterlogging in plants (Maxwell and Johnson 2000). However, decreases in CFR were not observed in experiment 1 of this study under WL treatments in the absence of PRR. With the level of root damage incurred by all lines in this study within the WL treatment, a subsequent reduction in photosynthetic potential was expected. The early growth stage of assessment and/or the controlled-environment phenotyping conditions used may have contributed toward the inability of chlorophyll fluorescence to capture root damage caused by waterlogging. Extending waterlogging may increase the likelihood of CFR detecting differences, as seen in experiment 2.

Genetic variation for waterlogging tolerance has historically been considered to be minimal in Australian chickpea material (Cowie et al. 1996; Palta et al. 2010). However, in this study we found root traits associated with waterlogging tolerance differed significantly between the three genotypes evaluated. Under waterlogged conditions, PRL of all three chickpea genotypes was reduced. However, the C. echinospermum BC 04067-81-2-1-1 was able to maintain a greater PRL under both WL and combined PRR:WL conditions over the $C$. arietinum chickpea genotypes Yorker and Rupali, which could be used as an indicator of superior PRR resistance. In support, greater PRL correlated to the level of PRR resistance using this rapid phenotyping method.

The ability of the C. echinospermum BC 04067-81-2-1-1 to maintain greater root length under waterlogged conditions may be linked to anaerobic respiration processes, higher root porosity, aerenchyma, and apoplastic barriers of the exo- and endo-dermal tissues. Genotypes that maintain roots at greater depth may have features that support anaerobic respiration or reduce radial oxygen loss, allowing distribution of oxygen to the lower root system (Kotula et al. 2017; Malik et al. 2001; Patrick and Delaune 1972). Based on the crossover of waterlogging tolerance and PRR resistance in C. echinospermum BC 04067-81-2-1-1, the apoplastic barrier of the exo- and endo-dermal tissues may be contributing to the higher levels of PRR
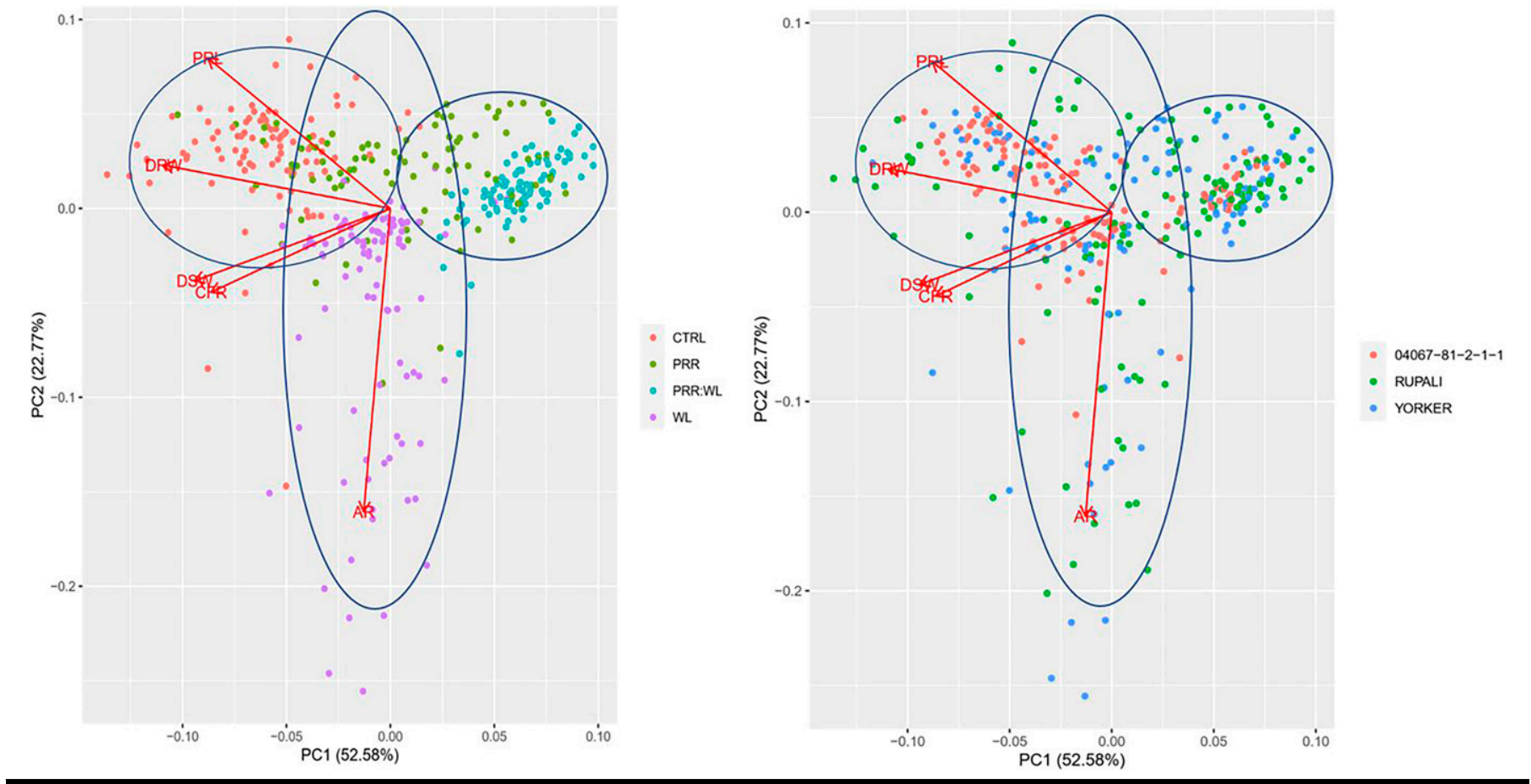

FIGURE 2

Experiment 1 principal component (PC) analyses indicating the treatment effect (left) and variety effect (right) across chickpea genotypes 04067-81-2-1-1 (resistant), Yorker (moderately susceptible), and Rupali (very susceptible). CTRL = control; PRR = inoculated with Phytophthora medicaginis; PRR:WL = inoculated with $P$. medicaginis with waterlogging; and $\mathrm{WL}=$ waterlogging treatment. Traits tested include primary root length, dry root weight, dry shoot weight, adventitious root count, and chlorophyll fluorescence ratio. 
TABLE 1

Effects of waterlogging and Phytophthora medicaginis inoculation on dry shoot weight (DSW), chlorophyll fluorescence ratio (CFR), dry root weight (DRW), primary root length (PRL), and adventitious root count (AR) for three chickpea genotypes ${ }^{\mathrm{a}}$

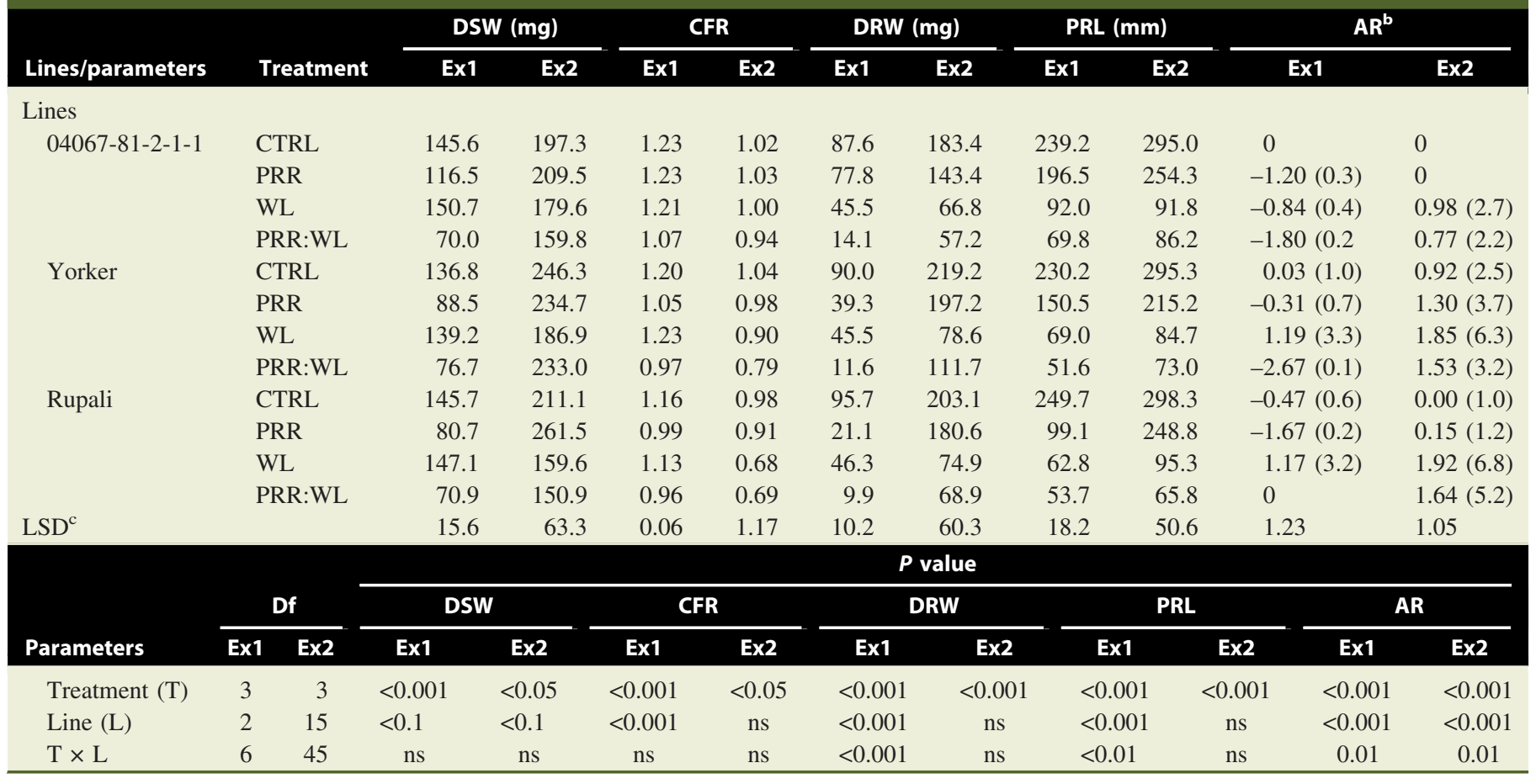

${ }^{a}$ Chickpea genotypes are wild Cicer back cross 04067-81-2-1-1 and Cicer arietinum Yorker and Rupali. Ex1 = experiment 1; Ex2 = experiment 2; and $\mathrm{ns}=$ not significant. Treatments: $\mathrm{CTRL}=$ control; $\mathrm{PRR}=$ Phytophthora medicaginis inoculated; $\mathrm{WL}=$ waterlogging; and $\mathrm{PRR}: \mathrm{WL}=P$. medicaginis inoculated and waterlogging in combination.

${ }^{\mathrm{b}}$ Back-transformed means in parentheses.

${ }^{\mathrm{c}} \mathrm{LSD}(\mathrm{T} \times \mathrm{L}), P=0.05$
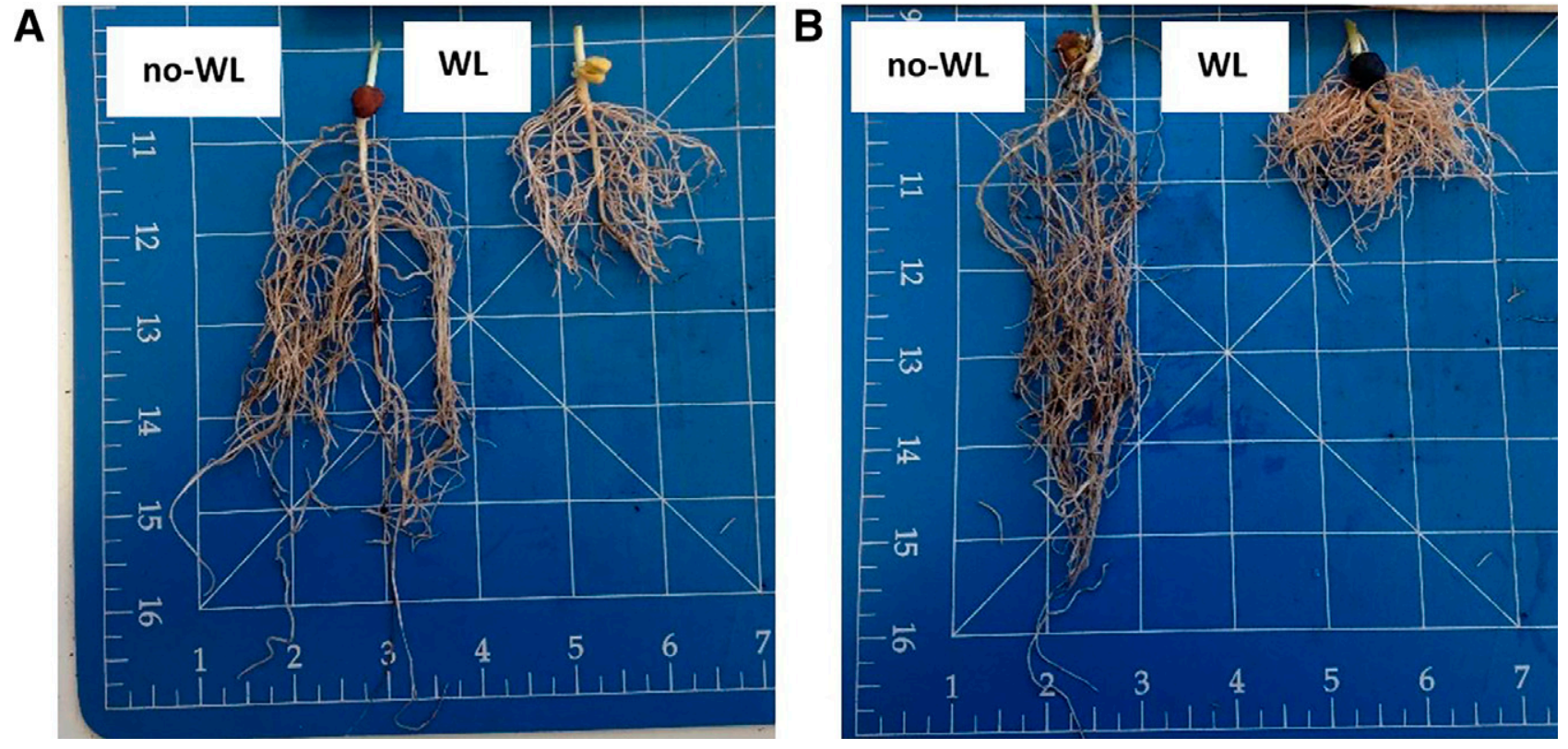

FIGURE 3

Root systems of representative seedlings not subjected (no-WL) or subjected to waterlogging (WL) for A, Phytophthora medicaginis (PRR)-resistant Cicer echinospermum backcross line 04067-81-2-1-1, and B, PRR-susceptible Rupali (C. arietinum). 
resistance observed, as previously demonstrated in soybean (Nguyen et al. 2012; Ranathunge et al. 2008).

Yorker and Rupali had an eightfold and threefold increase in early AR in the epicotyl and hypocotyl region compared with $C$. echinospermum BC 04067-81-2-1-1 under waterlogged conditions in experiments 1 and 2, respectively. Promoting secondary lateral and adventitious root growth at the basal region of the root and stem is a mechanism to escape waterlogging stress, because this region offers a reprieve from severe hypoxia or anoxia as the soil drains or remains free of flood water. This mechanism has been reported for several crops including wheat and rice (Kotula et al. 2017; Nishiuchi et al. 2012; Steffens and Rasmussen 2016). Chickpea is a hypogeal germinating species with an epicotyl that extends from the seed to the soil surface (Cubero 1987). Adventitious roots are not predetermined during early growth, instead developing later in response to a physiological requirement or stress (Steffens and Rasmussen 2016; Verstraeten et al. 2014). For adventitious roots to emerge unimpeded, the endodermis, cortex, exodermis, and epidermis must undergo remodeling through reactive oxygen species and peroxidase activity, essentially wounding the root. This may result in a temporary increase in permeability, as previously observed in onion (Peterson and Moon 1993). This permeability may be a contributing factor to increased PRR susceptibility under waterlogging conditions.

Understanding the implications of root plasticity and the extent of physiological damage identified within chickpea genotypes Yorker and Rupali under waterlogged conditions is an important piece of the puzzle when considering mechanisms of PRR resistance. Phytophthora species have a chemotaxic response to root exudates released by new root tissues, so zoospores are often found in high abundance at branch points and root apices (Erwin et al. 1983; Kuan and Erwin 1980; Suo et al. 2016; Tyler 2002). Hence, the rapid increase in adventitious root development in response to waterlogging in Yorker and Rupali could hasten pathogen entry and increase inoculum load, thereby increasing susceptibility to PRR. This could explain the observation that the PRR resistance rating of some varieties (such as Yorker) is often poorly expressed in seasons when infection occurs in combination with sustained or repeated periods of waterlogging.

However, in addition to branch points and root apices, stomata below the soil surface on the hypocotyl and epicotyl tissues are the preferred entry point for $P$. medicaginis zoospores (Dale and Irwin 1991). Under transient waterlogging conditions, stomatal conductance is increased in a bid to maintain leaf water potential in stressed plants (Bradford and Hsiao 1982). This stomatal closure does not appear to improve the survivability of chickpea to PRR under long-term waterlogging conditions. In addition, root tissues under waterlogging conditions suffer anoxic stress in which cell membranes undergo lipid peroxidation, and subsequently functional integrity is lost (Blokhina et al. 1999). This means that plants would have to rely on other biochemical responses for PRR resistance once they become infected. But under waterlogging conditions plants have reduced energy available to maintain secondary metabolic functions for a biochemical defense response (Blokhina et al. 1999). In chickpea, Dale and Irwin (1991) found that wounds from PRR-infected susceptible chickpea roots showed an increase in hyphal growth of $P$. medicaginis over resistant chickpea lines, further indicating that there are other biochemical or physiological responses reducing the colonization of $P$. medicaginis. Hence, both the mechanical barrier and maintaining normal metabolism are important for improving PRR resistance in chickpea through waterlogging tolerance.

\section{Conclusion}

Screening for waterlogging tolerance traits in chickpea offers an alternative method for improving PRR resistance. The $C$. echinospermum BC 04067-81-2-1-1, which has superior PRR resistance, maintained a greater rooting length and developed fewer adventitious roots under waterlogged conditions than the two other more susceptible genotypes. Increasing PRR resistance by means of reducing adventitious root development, or root vigor, should be considered carefully as it may impact yield potential. Searching for additional sources of waterlogging tolerance in Cicer spp. and extending these preliminary findings to examine responses in adult plants and field scenarios appear to be warranted. Further understanding of underlying PRR resistance mechanisms is essential to rapidly incorporate selected traits into adapted high-yielding chickpea cultivars to limit the losses from this disease. As shown in this study, adoption of an early stage waterlogging phenotypic screen as a proxy for PRR resistance within breeding programs could be beneficial. This would reduce the reliance on field studies, which are subject to rainfall variability or availability of irrigation. Phenotypic screening for waterlogging also enables the distinction of high and moderate levels of PRR resistance at the seedling stage with improved survivability and subsequent seed recuperation from adult plants.

\section{Acknowledgments}

The genotype BC 04067-81-2-1-1 was developed in DAN00065 and DAN00094 by Ted Knights. Kevin Moore and Sean Bithell developed the disease assessment and oospore production methods. Julie Hayes, Sean Bithell, Toni Petronaitis, and Tendo Mukasa Mugerwa assisted in proofing of this manuscript.

\section{Author Contributions}

N.D., K.H., and T.S. developed the phenotyping experiments. N.D. drafted the manuscript. All authors reviewed and revised the manuscript. K.H., T.S., and S.S. supervised the project. S.H. assisted with the experimental designs, principal component plots, and analysis of results for this manuscript.

\section{Literature Cited}

ABARES. 2018. Page 187 in: Australian Crop Report. Australian Bureau of Agricultural and Resource Economics and Sciences, Canberra, Australia.

Amalraj, A., Taylor, J., Bithell, S., Li, Y., Moore, K., Hobson, K., and Sutton, T. 2019a. Mapping resistance to Phytophthora root rot identifies independent loci from cultivated (Cicer arietinum L.) and wild (Cicer echinospermum PH Davis) chickpea. Theor. Appl. Genet. 132:1017-1033.

Amalraj, A., Taylor, J., and Sutton, T. 2019b. A hydroponics based high throughput screening system for Phytophthora root rot resistance in chickpea (Cicer arietinum L.). Plant Methods 15:82.

Arnell, N. W. 2004. Climate change and global water resources: SRES emissions and socio-economic scenarios. Glob. Environ. Change 14:31-52.

Bithell, S. L., Moore, K., Herdina, McKay, A., Harden, S., and Simpfendorfer, S. 2021. Phytophthora root rot of chickpea: Inoculum concentration and seasonally dependent success for qPCR based predictions of disease and yield loss. Australas. Plant Pathol. 50:91-103.

Blokhina, O. B., Fagerstedt, K. V., and Chirkova, T. V. 1999. Relationships between lipid peroxidation and anoxia tolerance in a range of species during post-anoxic reaeration. Physiol. Plant. 105:625-632.

Boye, J., Zare, F., and Pletch, A. 2010. Pulse proteins: Processing, characterization, functional properties and applications in food and feed. Food Res. Int. 43:414-431.

Bradford, K. J., and Hsiao, T. C. 1982. Stomatal behavior and water relations of waterlogged tomato plants. Plant Physiol. 70:1508-1513.

Brien, C. 2020. asremlPlus: Augments 'ASReml-R' in fitting mixed models and packages generally in exploring prediction differences. $\mathrm{R}$ package version 4.2-26. https://cran.r-project.org/web/packages/asremlPlus/index.html.

Brinsmead, R. B., Rettke, M. L., Irwin, J. A. G., Ryley, M. J., and Langdon, P. W. 1985. Resistance in chickpea to Phytophthora megasperma f. sp. medicaginis. Plant Dis. 69:504-506. 
Butler, D. 2020. asreml: Fits the Linear Mixed Model. R package version 4.1.0.143. https://www.vsni.co.uk/.

Chauhan, Y. 1987. Screening for tolerance to salinity and waterlogging: Case studies with pigeonpea and chickpea. Pages 93-103 in: Adaptation of Chickpea and Pigeonpea to Abiotic Stresses: Proceedings of the Consultants' Workshop, 19-21 December 1984. ICRISAT, Patancheru, India.

Chen, W., Sharma, H. C., and Muehlbauer, F. J., eds. 2011. Compendium of Chickpea and Lentil Diseases and Pests. American Phytopathological Society, St. Paul, MN

Cornelious, B., Chen, P., Chen, Y., De Leon, N., Shannon, J., and Wang, D. 2005. Identification of QTLs underlying waterlogging tolerance in soybean. Mol. Breed. 16:103-112.

Cowie, A. L., Jessop, R. S., and MacLeod, D. A. 1995. A study of waterlogging damage in Australian chickpea cultivars. Int. Chickpea Pigeonpea Newsl. 2: $22-23$

Cowie, A. L., Jessop, R. S., and MacLeod, D. A. 1996. Effects of waterlogging on chickpeas I. Influence of timing of waterlogging. Plant Soil 183:97-103.

Cubero, J. I. 1987. Morphology of chickpea. Pages 35-66 in: The Chickpea. M. C. Saxena and K. B. Singh, eds. CAB International, Wallingford, U.K.

Dale, M. L., and Irwin, J. A. G. 1991. Stomata as an infection court for Phytophthora megasperma f. sp. medicaginis in chickpea and a histological study of infection. Phytopathology 81:375-379.

Erwin, D. C., Bartnicki-Garcia, S., and Tsao, P. H., eds. 1983. Phytophthora: Its Biology, Taxonomy, Ecology, and Pathology. American Phytopathological Society, St. Paul, MN.

Gitelson, A. A., Buschmann, C., and Lichtenthaler, H. K. 1999. The chlorophyll fluorescence ratio F735/F700 as an accurate measure of the chlorophyll content in plants. Remote Sens. Environ. 69:296-302.

Hirabayashi, Y., Mahendran, R., Koirala, S., Konoshima, L., Yamazaki, D., Watanabe, S., Kim, H., and Kanae, S. 2013. Global flood risk under climate change. Nat. Clim. Chang. 3:816-821.

Jackson, M. B., and Colmer, T. D. 2005. Response and adaptation by plants to flooding stress. Ann. Bot. (Lond.) 96:501-505.

Knights, E. J., Açıkgöz, N., Warkentin, T., Bejiga, G., Yadav, S. S., and Sandhu, J. S. 2007. Area, production and distribution. Pages 167 to 178 in: Chickpea Breeding and Management. S. S. Yadav, R. J. Redden, W. Chen, and B. Sharma, eds. CABI, Wallingford, U.K.

Knights, E. J., Southwell, R. J., Schwinghamer, M. W., and Harden, S. 2008. Resistance to Phytophthora medicaginis Hansen and Maxwell in wild Cicer species and its use in breeding root rot resistant chickpea (Cicer arietinum L.). Aust. J. Agric. Res. 59:383-387.

Kotula, L., Schreiber, L., Colmer, T. D., and Nakazono, M. 2017. Anatomical and biochemical characterisation of a barrier to radial $\mathrm{O}_{2}$ loss in adventitious roots of two contrasting Hordeum marinum accessions. Funct. Plant Biol. 44:845-857.

Kuan, T. L., and Erwin, D. C. 1980. Predisposition effect of water saturation of soil on Phytophthora root rot of alfalfa. Phytopathology 70:981-986.

Maechler, M., Rousseeuw, P., Struyf, A., Hubert, M., and Hornik, K. 2019. Cluster: Cluster analysis basics and extensions. R package version, 2.1.0. https://cran.r-project.org/web/packages/cluster/cluster.pdf.

Malik, A. I., Colmer, T. D., Lambers, H., and Schortemeyer, M. 2001. Changes in physiological and morphological traits of roots and shoots of wheat in response to different depths of waterlogging. Funct. Plant Biol. 28:11211131.

Maxwell, K., and Johnson, G. N. 2000. Chlorophyll fluorescence-A practical guide. J. Exp. Bot. 51:659-668.

Murray, G. M., and Brennan, J. P. 2012. The Current and Potential Costs from Diseases of Oilseed Crops in Australia. Grain Research and Development Corporation, Kingston, Australia.

Nene, Y. L., Sheila, V. K., and Sharma, S. B. 1996. A World List of Chickpea and Pigeonpea Pathogens. ICRISAT, Patancheru, India.

Nguyen, V. T., Vuong, T. D., VanToai, T., Lee, J. D., Wu, X., Mian, M. A. R., Dorrance, A. E., Shannon, J. G., and Nguyen, H. T. 2012. Mapping of quantitative trait loci associated with resistance to Phytophthora sojae and flooding tolerance in soybean. Crop Sci. 52:2481-2493.

Nishiuchi, S., Yamauchi, T., Takahashi, H., Kotula, L., and Nakazono, M. 2012. Mechanisms for coping with submergence and waterlogging in rice. Rice (N. Y.) 5:2.

Palta, J. A., Ganjeali, A., Turner, N. C., and Siddique, K. H. M. 2010. Effects of transient subsurface waterlogging on root growth, plant biomass and yield of chickpea. Agric. Water Manage. 97:1469-1476.

Patrick, W., and Delaune, R. 1972. Characterization of the oxidized and reduced zones in flooded soil. Soil Sci. Soc. Am. J. 36:573-576.

Peterson, C. A., and Moon, G. J. 1993. The effect of lateral root outgrowth on the structure and permeability of the onion root exodermis. Bot. Acta 106: 411-418.

Pfender, W. F., Hine, R. B., and Stanghellini, M. E. 1977. Production of sporangia and release of zoospores by Phytophthora megasperma in soil. Phytopathology 67:657-663.

Ranathunge, K., Thomas, R. H., Fang, X. X., Peterson, C. A., Gijzen, M., and Bernards, M. A. 2008. Soybean root suberin and partial resistance to root rot caused by Phytophthora sojae. Phytopathology 98:1179-1189.

Samineni, S., Siddique, K. H., Gaur, P. M., and Colmer, T. D. 2011. Salt sensitivity of the vegetative and reproductive stages in chickpea (Cicer arietinum L.): Podding is a particularly sensitive stage. Environ. Exp. Bot. 71:260-268

Schwinghamer, M., Southwell, R., Moore, K., and Knights, E. 2011. Phytophthora root rot of chickpea. Pages 22-25 in: Compendium of Chickpea and Lentil Diseases and Pests. W. Chen, H. C. Sharma, and F. J. Muehlbauer, eds. American Phytopathological Society, St. Paul, MN.

Steffens, B., and Rasmussen, A. 2016. The physiology of adventitious roots. Plant Physiol. 170:603-617.

Stolzy, L. H., and Sojka, R. E. 1984. Effects of flooding on plant disease. Pages 221-264 in: Flooding and Plant Growth. T. T. Kozlowski, ed. Academic Press, San Diego, CA

Suo, B., Chen, Q., Wu, W., Wu, D., Tian, M., Jie, Y., Zhang, B., and Wen, J. 2016. Chemotactic responses of Phytophthora sojae zoospores to amino acids and sugars in root exudates. J. Gen. Plant Pathol. 82:142-148.

Tyler, B. M. 2002. Molecular basis of recognition between Phytophthora pathogens and their hosts. Annu. Rev. Phytopathol. 40:137-167.

Van West, P., Appiah, A. A., and Gow, N. A. 2003. Advances in research on oomycete root pathogens. Physiol. Mol. Plant Pathol. 62:99-113.

Verstraeten, I., Schotte, S., and Geelen, D. 2014. Hypocotyl adventitious root organogenesis differs from lateral root development. Front. Plant Sci. 5:495. 